\title{
Molecular and Epidemiological Characteristics of Norovirus Associated with Community-Based Sporadic Gastroenteritis Incidents and Norovirus Outbreaks in Victoria, Australia, 2002-2007
}

\author{
Leesa Bruggink Rizmina Sameer John Marshall \\ Gastroenteritis Section, Victorian Infectious Diseases Reference Laboratory, North Melbourne, Vic., Australia
}

\section{Key Words}

Sporadic gastroenteritis - Gastroenteritis outbreak •

Norovirus genotype

\begin{abstract}
Objectives: The molecular and epidemiological features of community-based norovirus-associated sporadic gastroenteritis incidents (NASGIs) are poorly understood. This study examined these features and compared the findings with studies of community-based and institutional norovirus-associated gastroenteritis outbreaks (NAGOs). Methods: Fecal specimens from NASGIs and NAGOs that occurred in Victoria, Australia (2002-2007) were tested for norovirus by reverse transcription-polymerase chain reaction methodology. Norovirus genotype was determined by nucleotide sequence analysis. Results: 106 community-based NASGIs, 116 community-based NAGOs and 902 institutional NAGOs were identified. The mean age and gender ratio of individuals associated with community-based NASGls and communitybased NAGOs were similar but differed from that found for institutional NAGOs. Although GIl.4 was the predominant genotype associated with all three incident types, the mix of genotypes was similar for community-based NASGls and community-based NAGOs but that for institutional NAGOs was different. All three incident types had a similar seasonal
\end{abstract}

periodicity due to the pronounced seasonal periodicity of GIl.4 incidents. Conclusions: The molecular and epidemiological features of noroviruses associated with communitybased NASGIs and community-based NAGOs are similar but are different from those found for institutional NAGOs.

Copyright $\odot 2010$ S. Karger AG, Basel

\section{Introduction}

The noroviruses are single-stranded positive-sense RNA viruses classified as the genus Norovirus within the family Caliciviridae. Noroviruses are classified into five genogroups of which two, genogroup I (GI) and genogroup II (GII), are the most commonly found in humans. Within each genogroup, a number of genotypes have been identified [1].

Noroviruses are now recognized as one of the major causes of viral gastroenteritis [1]. Norovirus incidents in humans can be considered to fall into two groupings, sporadic incidents (norovirus-associated sporadic gastroenteritis incident or 'NASGI') and outbreaks (norovirus-associated gastroenteritis outbreak or 'NAGO') [1]. Depending on the setting of the outbreak, NAGOs can be described as 'institutional' NAGOs or as 'communitybased' NAGOs.

\section{KARGER}

Fax +4161306 1234 E-Mail karger@karger.ch www.karger.com
(C) 2010 S. Karger AG, Basel

0300-5526/10/0533-0167\$26.00/0

Accessible online at:

www.karger.com/int
Dr. J.A. Marshall

Victorian Infectious Diseases Reference Laboratory

10 Wreckyn Street

North Melbourne, Vic. 3051 (Australia)

Tel. +61 39342 2678, Fax +61 39342 2660,E-Mail john.marshall@mh.org.au 
Most literature studies on NASGIs relate to children who have presented at a hospital or clinic with gastroenteritis [2]. Studies relating to NASGIs for adults presenting at hospitals or clinics are documented but less common [2]. Least common of all are community-based studies of NASGIs in adults and children who have not necessarily presented at a hospital or clinic $[2,3]$, although there is evidence such community-based illness is an important cause of morbidity in the population [3]. This study analyzed such community-based NASGIs.

The precise relationship between norovirus genotype and the setting of a NASGI or NAGO is not fully understood, but there is a growing body of evidence which indicates some norovirus genotypes may be more commonly associated with some settings than others. For example, Fankhauser et al. [4] noted that certain GII clusters were more common in outbreaks in nursing homes than outbreaks in other settings in a study of norovirus outbreaks in the USA. Gallimore et al. [5] found that GI noroviruses were more common in outbreaks in cruise ships than in UK hospitals and other semi-closed institutions. Gallimore et al. [6] found that GI noroviruses were more common in community-based outbreaks than in hospital and nursing home outbreaks in England. Bon et al. [7] found differences in the incidence of certain norovirus genotypes when outbreaks in different settings were compared in France. There is also evidence that the mix of genotypes associated with community-based NASGIs compared to NAGOs in general may be different [8]. The detailed relationship between the mix of norovirus genotypes associated with community-based NASGIs, community-based NAGOs and institutional NAGOs, however, is unknown.

The aim of the current study was to establish the molecular and epidemiological features of community-based NASGIs and to compare and contrast the findings with those associated with community-based and institutional NAGOs, respectively. Material obtained in Victoria, Australia, in the period 2002-2007, was utilized for the study.

\section{Materials and Methods}

Definition of Sporadic Gastroenteritis Incidents and Gastroenteritis Outbreaks

For the purposes of this study, a community-based sporadic gastroenteritis incident was defined as one which involved 3 or fewer individuals who experienced gastroenteritis and made a complaint to a municipal health authority. A rationale for this definition of a sporadic gastroenteritis incident is given in the
'Discussion'. In all cases, the complainant suggested the gastroenteritis was related to consumption of food within the jurisdiction of a particular municipality. Documentation of sporadic gastroenteritis incidents was provided by the relevant municipal council.

During the course of the study (2002-2007), 445 specimens were received from 398 sporadic gastroenteritis incidents. 355 involved a single individual, 39 involved 2 individuals and 4 involved 3 individuals. Only sporadic gastroenteritis incidents which occurred in the state of Victoria were included in the study. The date of the incident was taken as the date of the earliest specimen collected or, if this date was not available, the earliest date a specimen was received for testing. The incidents were those for which specimens were sent for testing to the Victorian Infectious Diseases Reference Laboratory, which is the main public health laboratory for viral identification in the state of Victoria, Australia.

An outbreak of gastroenteritis was defined as an incident, apparently associated with a common event or location, in which 4 or more individuals had symptoms of gastroenteritis. A rationale for this definition is given in the 'Discussion'. The outbreaks included in this study were those for which specimens were sent to the Victorian Infectious Diseases Reference Laboratory for testing. Only outbreaks which occurred in the state of Victoria were included in the study. During the course of the study (2002-2007), 8,507 specimens from 1,495 outbreaks were received for testing.

The date of the outbreak was taken as the onset date if provided. If this was unavailable, the date taken was the date when the outbreak was first notified to the Department of Human Services or the earliest date of collection of a specimen for testing. Outbreak documentation (including the setting of the outbreak) and management were carried out by staff of the Department of Human Services and/or staff at the affected setting.

NAGOs were classified into two groups, 'institutional' and 'community based'. An institutional setting was taken to be one where inmates were confined to the setting, usually for longer periods. A community-based setting was one where people joined together voluntarily, usually for shorter periods. In essence, institutional settings corresponded to 'healthcare' settings (but also included prisons), whereas community-based settings corresponded to 'non-healthcare' settings.

Institutional settings of NAGOs in this study included agedcare facilities, disabled care facilities, early parenting centers, hospitals, hospitals-geriatric ward, hospitals-pediatric ward, hospitals-plastic surgery unit, hospitals-psychiatric ward, hospitalsrehabilitation unit, a palliative care facility and prisons. Of the 902 institutional NAGOs identified, 722 (80.0\%) were associated with settings associated with the elderly (i.e. the categories agedcare facilities and hospitals-geriatric ward). Community-based settings included barracks, camps, camp-adult, camp-school, child care centers, children's activity centers, elderly activity centers, gatherings, a navy base, restaurants and suspect food.

\section{Fecal Processing and RNA Extraction}

Fecal specimens were prepared as a $20 \%$ (vol/vol) suspension in Hanks' complete balanced salt solution, the suspension vigorously shaken, then clarified by centrifugation [9]. The clarified fluid was then collected for RNA extraction. RNA extraction, prior to open reading frame (ORF) 1 reverse transcription-polymerase chain reaction (RT-PCR), was then carried out using ei- 
ther the manual Qiagen QIAamp viral RNA mini-kit cat. No. 52906 (Qiagen GmbH, Hilden, Germany) [10] or the Corbett (Corbett Robotics Pty., Ltd., Eight Mile Plains, Qld., Australia) automated nucleic acid extraction procedure [10].

\section{Norovirus ORF 1 RT-PCR}

ORF 1 RT-PCR testing for norovirus was carried out essentially as given by Yuen et al. [11]. The RT-PCR detection method utilized was a two-round heminested multiplex procedure which both detects and discriminates between GI and GII noroviruses [11]. The method is highly sensitive and specific [11, 12], and molecular studies in this laboratory [8] have further confirmed the assay detects a broad range of norovirus genotypes. The assay incorporates a second round PCR step which serves as both a confirmatory test and as a booster step for enhanced sensitivity. Specimens were only taken as positive if they were positive in the first and second rounds of the PCR or if they were positive in the second round only.

\section{Norovirus ORF 1 Nucleotide Sequencing and Phylogenetic}

Analysis

Genotype definition of all NAGOs initially involved sequencing analysis of one norovirus specimen, chosen without bias, from each outbreak where a positive norovirus specimen was identified. If both GI and GII specimens were identified in a NAGO, then an attempt was made to sequence one of each. Genotype definition for all NASGIs was carried out as for NAGOs except that all norovirus-positive specimens in an incident were sequenced where more than one norovirus-positive specimen was identified in that incident.

The second-round PCR product of the ORF 1 RT-PCR test procedure was purified and sequenced essentially as given previously [3]. A region of 440 nucleotides within the RNA polymerase region, which corresponded to nucleotides 4,484-4,923 of Camberwell virus (AF145896), was then utilized for phylogenetic analysis and genotype definition.

MacVector software versions 7.2 and 8.0 (Oxford Molecular Limited, Madison, Wisc., USA) were used for analysis of nucleotide sequences. Phylogenetic analysis was carried out using the software PHYLIP, versions 3.572, 3.65 or 3.68 [13]. The distancebased (Kimura matrix) neighbor-joining method was the principal algorithm used for analysis (functions DNADIST and NEIGHBOR), and the trees were constructed with the software Treeview version 1.5 or FigTree version 1.2.2. The robustness of the neighbor-joining tree generated was assessed by bootstrap resampling and compared with trees constructed using alternate algorithms as described previously [3]. Separate ORF 1 phylogenetic trees were generated for all NASGIs and, for each year of the study, for NAGOs (results not shown).

Genotypes identified in this study were classified after comparison with documented strains ('reference strains'). A broad range of reference strains, essentially as given by Bruggink and Marshall [14], were used for genotype definition.

\section{Statistical Analysis}

The statistical significance of differences between average ages for NASGIs, community-based NAGOs and institutional NAGOs was determined by the two-tailed large-sample significance test for a difference between means [15] and the significance then calculated following Bonferroni's method [16]. The statistical significance of differences in the proportions of individual genotypes was determined by the $\chi^{2}$ test, and the significance then calculated following Bonferroni's method [16].

The statistical significance of differences in gender ratios or genotype distribution was determined by the partitioning of $\chi^{2}$ procedure [17]. Differences for partitioning of $\chi^{2}$ were taken to be significant when $\mathrm{p}<0.05$.

\section{Results}

\section{General Comparison of the Features of NASGIs and NAGOs}

During the period 2002-2007, 445 fecal specimens from 398 community-based sporadic gastroenteritis incidents were tested for norovirus. Norovirus was detected in 116 specimens, which corresponded to at least one positive specimen in each of 106 incidents ('NASGIs'). Ninety-one of these NASGIs involved one individual and 15 involved 2 individuals, where one or both were positive for norovirus. None involved 3 individuals. GI norovirus only was detected in 14 (13.2\%) NASGIs and GII norovirus only was detected in $92(86.8 \%)$ NASGIs. There were no NASGIs with both GI and GII norovirus detected.

During the period 2002-2007, 8,507 fecal specimens from 1,495 gastroenteritis outbreaks were tested for norovirus. Norovirus was detected in 4,561 specimens. This corresponded to at least one norovirus-positive specimen in 1,018 outbreaks ('NAGOs'). Of these, GI norovirus only was detected in 42 (4.1\%) NAGOs, GII norovirus only in 972 (95.5\%) NAGOs and both GI and GII in 4 (0.4\%) NAGOs.

Of the 1,018 NAGOs identified, 116 were communitybased and 902 were institutional. For the communitybased NAGOs, GI norovirus only was detected in 16 (13.8\%), GII norovirus only in 99 (85.3\%) and both GI and GII in one $(0.9 \%)$. For the institutional NAGOs, GI norovirus only was detected in 26 (2.9\%), GII norovirus in 873 (96.8\%) and both GI and GII in 3 (0.3\%).

The ages of individuals associated with the categories NASGI, community-based NAGO and institutional NAGO are given in table 1 . It was found that the mean age of individuals associated with NASGIs was not significantly different from that for community-based NAGOs ( $p>0.10)$, but the mean age for institutional NAGOs was significantly different from that for NASGIs and community-based NAGOs ( $\mathrm{p}<0.001$ in both cases).

Information on gender ratios in NAGOs and NASGIs also indicated the ratios were similar for the communitybased NASGIs and community-based NAGOs, but were different in institutional NAGOs. In particular, for all no- 
Table 1. Ages (mean \pm SD) of norovirus-positive individuals associated with NASGIs and NAGOs

\begin{tabular}{lcc}
\hline Category & Age, years & $\begin{array}{l}\text { Individuals of } \\
\text { known age }\end{array}$ \\
\hline NASGIs & $34.1 \pm 17.2$ & 105 \\
Community-based NAGOs & $31.1 \pm 24.4$ & 404 \\
Institutional NAGOs & $80.1 \pm 15.3$ & 3,803 \\
\hline
\end{tabular}

rovirus-positive individuals of known gender, the percentages of norovirus-positive males and females, respectively, were 55.2 and $44.8 \%$ for NASGIs, 47.0 and $53.0 \%$ for community-based NAGOs and 28.3 and $71.7 \%$ for institutional NAGOs. The gender ratios for NASGIs and community-based NAGOs were not significantly different from each other $\left(p>0.10\right.$, partitioning of $\left.\chi^{2}\right)$ but both were significantly different from the gender ratio for institutional NAGOs $\left(\mathrm{p}<0.001\right.$, partitioning of $\left.\chi^{2}\right)$.

\section{Comparison of Norovirus Genotypes Associated with}

\section{NASGIs and NAGOs}

Norovirus genotypes associated with NASGIs, community-based NAGOs and institutional NAGOs are given in table 2. It should be noted that in the 10 NASGIs where 2 norovirus-positive specimens were identified, the sequences were identical in 9 of the 10 NASGIs; in the 10th NASGI, only one of the 2 specimens could be sequenced.

It can be seen from table 2 that the breakdown of the incidence of different genotypes associated with NASGIs and community-based NAGOs is similar, whereas that for institutional NAGOs is different. The distributions of genotypes for NASGIs and community-based NAGOs were not significantly different from each other $(p>0.70$, partitioning of $\chi^{2}$ ), but the distribution of genotypes for both of these categories was significantly different from that for institutional NAGOs $(\mathrm{p}<0.001$, partitioning of $\chi^{2}$ ). Separate $\chi^{2}$ tests for each group of genotypes indicated that the proportions of the principal genotypes or genotype combinations GII.4, GIIb and GI.2 plus GI.3b in institutional NAGOs were significantly different from those in NASGIs and community-based NAGOs ( $\mathrm{p}<$ 0.001, $\chi^{2}$ test).

Figure 1 gives the seasonal periodicity of norovirus genotypes associated with NASGIs (fig. 1a), communitybased NAGOs (fig. 1b) and institutional NAGOs (fig. 1c). In all three categories of incident, GII.4 was the predominant genotype detected and GII.4 had a marked season-
Table 2. Genotypes found in NASGIs, community-based NAGOs and institutional NAGOs, 2002-2007

\begin{tabular}{|c|c|c|c|c|c|c|}
\hline \multirow{3}{*}{$\begin{array}{l}\text { Geno- } \\
\text { type }\end{array}$} & \multicolumn{2}{|c|}{ NASGIs } & \multicolumn{4}{|c|}{ NAGOs } \\
\hline & \multirow[t]{2}{*}{$\mathrm{n}$} & \multirow[t]{2}{*}{$\%$} & \multicolumn{2}{|c|}{ community } & \multicolumn{2}{|c|}{ institutional } \\
\hline & & & $\mathrm{n}$ & $\%$ & $\mathrm{n}$ & $\%$ \\
\hline GI.2 & 7 & 6.9 & 7 & 6.4 & 5 & 0.6 \\
\hline GI.3b & 3 & 3.0 & 3 & 2.8 & 4 & 0.4 \\
\hline GI.4 & 1 & 1.0 & 4 & 3.7 & 14 & 1.6 \\
\hline GI.?a ${ }^{1}$ & 0 & 0.0 & 0 & 0.0 & 1 & 0.1 \\
\hline GI.?b ${ }^{1}$ & 1 & 1.0 & 1 & 0.9 & 2 & 0.2 \\
\hline GI.?c $c^{1}$ & 1 & 1.0 & 0 & 0.0 & 0 & 0.0 \\
\hline GII.1 & 0 & 0.0 & 0 & 0.0 & 1 & 0.1 \\
\hline GII.3a & 0 & 0.0 & 1 & 0.9 & 1 & 0.1 \\
\hline GII.3c & 0 & 0.0 & 0 & 0.0 & 1 & 0.1 \\
\hline GII.4 & 73 & 72.3 & 74 & 67.9 & 839 & 93.6 \\
\hline GIIb & 15 & 14.9 & 18 & 16.5 & 25 & 2.8 \\
\hline GI.2/GII.4 & 0 & 0.0 & 0 & 0.0 & 2 & 0.2 \\
\hline GI.4/GII.4 & 0 & 0.0 & 1 & 0.9 & 1 & 0.1 \\
\hline Total & 101 & 100.0 & 109 & 100.0 & 896 & 100.0 \\
\hline
\end{tabular}

Five unsequenced NASGIs and 13 unsequenced NAGOs (7 community-based, 6 institutional) are not included in the table.

${ }^{1}$ All three clusters fell separately on a tree (maximum amino acid identity between the strains was only $95 \%$, whereas $97.9 \%$ or greater was required for strains to be classified in the same cluster). BLAST searches did not reveal any known genotype classification for these three clusters.

al periodicity. The seasonal pattern of the dominant GII.4 genotype is reflected in the general seasonal trend for all three categories of incident.

\section{Discussion}

There appears to be general agreement that norovirusassociated gastroenteritis incidents can be divided into two types: NAGOs and NASGIs [1]. However, precise definitions which discriminate between NAGOs and NASGIs are lacking. The key question appears to be how many ill individuals or specimens from ill individuals are necessary for a gastroenteritis cluster to be considered a NAGO rather than a NASGI.

There have been many proposals as to the exact number of ill individuals and/or specimens available for testing from a particular gastroenteritis cluster for that cluster to be defined as a NAGO. For example, Lopman et al. [18] considered any cluster of 2 or more ill individuals as constituting a NAGO. Green et al. [19], who examined NAGOs 
Fig. 1. The seasonal periodicity of norovirus genotypes associated with NASGIs (a), community-based NAGOs (b) and institutional NAGOs (c) in Victoria, Australia, 2002-2007.

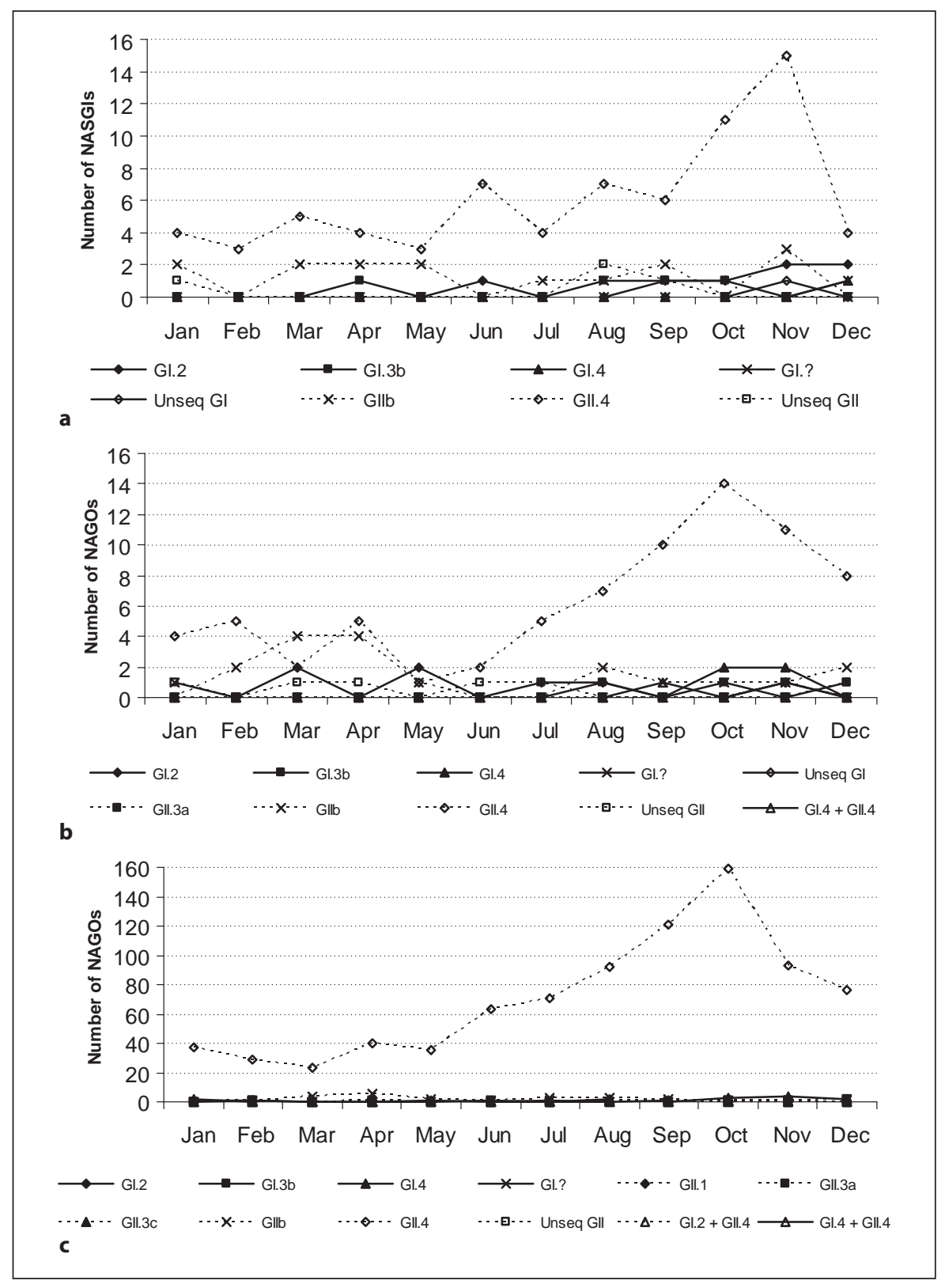

associated with nursing homes for the elderly, considered 3 or more ill individuals as being the minimum number for the cluster to be considered an outbreak. Fankhauser et al. [4], in an examination of NAGOs in the USA, indicated that only those outbreaks for which 4 or more specimens were available for testing were to be included in their study. Thus, it can be considered that a cluster of 4 or more ill individuals represents a NAGO, and it can be argued that a cluster of 3 or fewer ill individuals represents a NASGI.

The chief finding of the current study is that the mean age of individuals, gender ratios and genotypic mix as- sociated with community-based NASGIs and community-based NAGOs are similar to each other, and differ from the mean age of individuals, gender ratio and genotypic mix associated with institutional NAGOs. Institutional NAGOs were detected chiefly in aged-care facilities so the differing age and gender for community-based NASGIs and NAGOs compared to institutional NAGOs must reflect the older demographic associated with institutional outbreaks. The differing genotype mix associated with community-based NASGIs and NAGOs and institutional NAGOs is more difficult to explain, but the 
data suggest norovirus genotypes GIIb and GI.2 plus GI.3b, in particular, have a different epidemiology to that of GII.4.

The chief point of similarity of the NASGIs and the two types of NAGOs was that the seasonal periodicity of all three was essentially the same. This was due to the preponderance of the GII.4 norovirus genotype, which occurred with a marked seasonal periodicity.

Studies on NASGIs exactly comparable to the current study appear to be rare, presumably because collection of community-based gastroenteritis specimens from individuals who have not presented at a hospital or medical clinic can be difficult. However, a similar study was carried out in this laboratory previously [3]. That study focused on a specific group of individuals in a limited geographic area of Melbourne, Vic., and monitored community-based gastroenteritis over the period from September 1997 to February 1999. Based on the reference strains utilized in the GII phylogenetic analysis, it can be determined that the only GII genotypes identified were GII.1, GII.3 (two clusters) and GII.4. In contrast, in the current study, the only GII genotypes detected were GII.4 and GIIb. In both studies, the predominant norovirus geno- type detected was GII.4, but the findings suggest that the mix of norovirus genotypes circulating in the community in Victoria may have changed over the period from 1997 to 2007. It is particularly notable that the GIIb norovirus genotype, which was relatively common in the current study, was not detected in the earlier study.

Community-based norovirus-associated sporadic gastroenteritis is an important source of morbidity [3] but has been little studied. The findings of the current study indicate that the mix of norovirus genotypes associated with community-based gastroenteritis, whether sporadic incidents or outbreaks, is essentially the same but is different from that found in institutional outbreaks. The findings suggest that the mix of norovirus genotypes associated with community-based sporadic gastroenteritis incidents in Victoria may have changed over the period from 1997 to 2007, with the GIIb genotype emerging as increasingly important. Further studies are needed to monitor whether the mix of norovirus genotypes associated with community-based sporadic gastroenteritis incidents will undergo change in the coming years and whether the findings of the current study are borne out in similar studies in other parts of the world.

\section{References}

1 Marshall JA, Bruggink LD: Laboratory diagnosis of norovirus. Clin Lab 2006;52:571581.

2 Patel MM, Widdowson M-A, Glass RI, Akazawa K, Vinje J, Parashar UD: Systematic literature review of role of noroviruses in sporadic gastroenteritis. Emerg Infect Dis 2008; 14:1224-1231.

3 Marshall JA, Hellard ME, Sinclair MI, Fairley CK, Cox BJ, Catton MG, Kelly H, Wright PJ: Incidence and characteristics of endemic Norwalk-like virus-associated gastroenteritis. J Med Virol 2003;69:568-578.

4 Fankhauser RL, Monroe SS, Noel JS, Humphrey CD, Bresee JS, Parashar UD, Ando T, Glass RI: Epidemiologic and molecular trends of "Norwalk-like viruses" associated with outbreaks of gastroenteritis in the United States. J Infect Dis 2002;186:1-7.

$\checkmark 5$ Gallimore CI, Richards AF, Gray JJ: Molecular diversity of noroviruses associated with outbreaks on cruise ships: comparison with strains circulating within the UK. Commun Dis Public Health 2003;6:285293.

6 Gallimore CI, Green J, Lewis D, Richards AF, Lopman BA, Hale AD, Eglin R, Gray JJ, Brown DWG: Diversity of noroviruses cocirculating in the North of England from 1998 to 2001. J Clin Microbiol 2004;42:13961401 .
-

Bon F, Ambert-Balay K, Giraudon H, Kaplon J, Le Guyader S, Pommepuy M, Gallay A Vaillant V, de Valk H, Chikhi-Brachet R, Flahaut A, Pothier P, Kohli E: Molecular epidemiology of caliciviruses detected in sporadic and outbreak cases of gastroenteritis in France from December 1998 to February 2004. J Clin Microbiol 2005;43:4659-4664.

$\checkmark 8$ Marshall JA, Dimitriadis A, Wright PJ: Molecular and epidemiological features of norovirus-associated gastroenteritis outbreaks in Victoria, Australia in 2001. J Med Virol 2005; 75:321-331.

9 Witlox KJ, Karapanagiotidis T, Bruggink LD, Marshall JA: The effect of fecal turbidity on norovirus detection by reverse transcriptase polymerase chain reaction. Diagn $\mathrm{Mi}$ crobiol Infect Dis 2008, Epub ahead of print.

10 Witlox KJ, Nguyen TN, Bruggink LD, Catton MG, Marshall JA: A comparative evaluation of the sensitivity of two automated and two manual nucleic acid extraction methods for the detection of norovirus by RT-PCR. J Virol Methods 2008;150:70-72.

11 Yuen LKW, Catton MG, Cox BJ, Wright PJ, Marshall JA: Heminested multiplex reverse transcription-PCR for detection and differentiation of Norwalk-like virus genogroups 1 and 2 in fecal samples. J Clin Microbiol 2001;39:2690-2694.
12 Dimitriadis A, Bruggink LD, Marshall JA: Evaluation of the Dako IDEIA norovirus EIA assay for detection of norovirus using faecal specimens from Australian gastroenteritis outbreaks. Pathology 2006;38:157-165.

13 Felsenstein J: PHYLIP - Phylogeny Inference Package (Version 3.2). Cladistics 1989;5: 164-166.

14 Bruggink LD, Marshall JA: Molecular and epidemiological features of GIIb norovirus outbreaks in Victoria, Australia, 2002-2005. J Med Virol 2009;81:1652-1660.

15 Hays WL: Statistics for Psychologists. New York, Holt Rinehart and Winston, 1963, pp 316-319.

16 Fisher LD, van Belle G: Biostatistics: A Methodology for the Health Sciences. New York, John Wiley \& Sons, 1993, pp 611-613.

17 Agresti A: Categorical Data Analysis. New York, John Wiley \& Sons, 1990, pp 50-53.

18 Lopman BA, Adak GK, Reacher MH, Brown DWG: Two epidemiologic patterns of Norovirus outbreaks: surveillance in England and Wales, 1992-2000. Emerg Infect Dis 2003;9: $71-77$.

19 Green KY, Belliot G, Taylor JL, Valdesuso J, Lew JF, Kapikian AZ, Lin F-YC: A predominant role for Norwalk-like viruses as agents of epidemic gastroenteritis in Maryland nursing homes for the elderly. J Infect Dis 2002;185:133-146. 